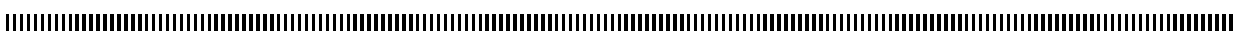

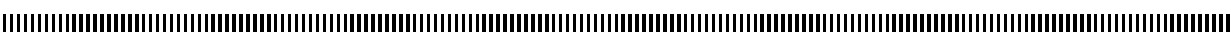
| |

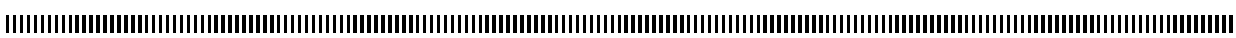
| |

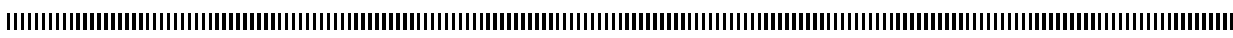

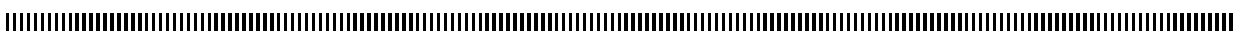
|

\title{
On the representation of L-M algebra by intuitionistic fuzzy subsets
}

\section{Sur la représentation d'algèbre de M-L par des sous-ensembles flous intuitionnistes}

\author{
Zedam Lemnaouar and Amroune Abdelaziz \\ Laboratory of Pure and Applied Mathematics, \\ M'sila University, \\ P.O. Box 166 Ichbilia, M'sila 28105, \\ Algeria. \\ lzedam@yahoo.fr and aamrounedz@yahoo.fr
}

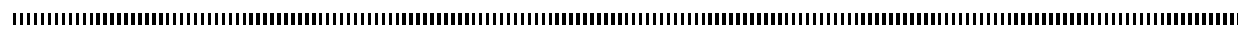

ABSTRACT. In this paper, we introduce the notions of intuitionistic weak $\alpha$-cut and intuitionistic strong $\alpha$-cut of intuitionistic fuzzy subset on a universe $X$. These notions lead us to show that the set $\operatorname{IF}(X)$ of all intuitionistic fuzzy subsets on a universe $X$ can be equipped with a structure of involutive $\theta$-valued Lukasiewicz-Moisil algebra. Conversely, we show that every involutive $\theta$-valued Lukasiewicz-Moisil algebra can be embedded into an algebra of intuitionistic fuzzy subsets.

RÉSUMÉ. Dans ce travail nous introduisons les notions des $\alpha$-coupes et des $\alpha$-coupes strictes d'un sous-ensemble flou intuitionniste d'un référentiel $X$. A l'aide de ces notions, nous montrons que l'ensemble $I F(X)$ des sous-ensembles flous intuitionnistes d'un référentiel $X$ admet une structure d'algèbre de Moisil-£ukasiewicz $\theta$-valente involutive. Réciproquemet, nous démontrons que toute algèbre de Moisil-£ukasiewicz $\theta$-valentes involutive se plonge dans une algèbre des sous-ensembles flous intuitionnistes involutives.

KEYWORDS : Lukasiewicz-Moisil algebras, Representation theorems, Intuitionistic fuzzy subsets.

MOTS-CLÉS : Algèbres de Moisil-Lukasiewicz, Théorèmes de représentation, Sous-ensembles flous intuitionnistes.

2000 Mathematics Subject Classification. 03G10, 03G20, 03E72, 08A72, $03 F 55$.

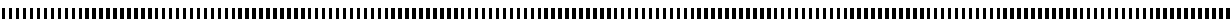

Volume 4 - 2006, pages 72 à 85 - ARIMA

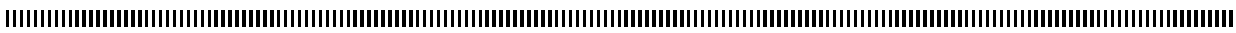

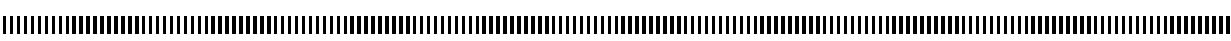

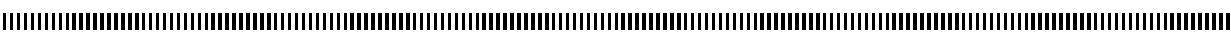
|

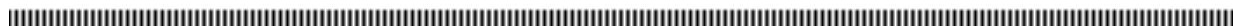




\section{Introduction}

In 1941 [11], Moisil introduced the notion of Lukasiewicz-Moisil algebra under the name Lukasiewician algebras, as an algebraic counterpart of the Lukasiewicz manyvalued logics. The theory of Lukasiewicz-Moisil algebras has been developed both as a tool for studying certain non-classical logics and as an algebraic theory having its own interest ; besides, now it is considered as one of the fundamental formalizations of fuzzy logic. For more details the reader is referred e.g. to [7, 8, 13, 14, 15].

After the introduction of the concept of fuzzy sets by Zadeh [21] in 1965, several mathematicians introduced and studied fuzzy substructures of many algebraic structures. For example, Negoita and Ralescu [16] introduced the notion of cuts of a fuzzy subset. Moisil [15] used these cuts and proving that the set of all fuzzy subsets over the universe of discourse $X$ can be equipped with a structure of $\theta$-valued Lukasiewicz-Moisil algebra. Conversely, Ponasse [17] proved that every $\theta$-valued Lukasiewicz-Moisil algebra can be embedded in an algebra of fuzzy subsets. In [17] Ponasse has also studied the representability of involutive $\theta$-valued Lukasiewicz-Moisil algebra as an algebra of fuzzy subsets equipped with an order-reversing involution. This problem was also investigated in $[1,9,10,18]$.

In another direction, Atanassov [2,5] introduced another fuzzy object, called intuitionistic fuzzy subset (briefly IFS) as a generalization of the concept of fuzzy subset. Certainly fuzzy subsets are intuitionistic fuzzy subsets but not conversely.

As outlined in $[3,6]$, fuzzy subset theory is not appropriate to deal with such problems; rather IFS theory is more suitable. The problems which are dealt with fuzzy subset theory can be well dealt with IFS theory. In the present paper, we give an application of IFSs in the theory of Lukasiewicz-Moisil algebras. Specifically, we prove that every involutive $\theta$-valued Lukasiewicz-Moisil algebra can be embedded into an algebra of intuitionistic fuzzy subsets. Also the set $I F(X)$ of all intuitionistic fuzzy subsets on a universe $X$ represents the involutive $\theta$-valued Lukasiewicz-Moisil algebra can be equipped with a structure of involutive $\theta$-valued Lukasiewicz-Moisil algebra. In order to give this representation theorems, we introduce the notions of intuitionistic weak $\alpha$-cut and intuitionistic strong $\alpha$-cut of intuitionistic fuzzy subset on a universe $X$.

In Section 2, we recall the notions of an involutive $\theta$-valued Lukasiewicz-Moisil algebra $[12,13,14]$ and give some notations, definitions and results on which our work in this paper is based. Afterwards, we do the same with the intuitionistic fuzzy subsets. We end this section by introducing the intuitionistic weak $\alpha$-cut and intuitionistic strong $\alpha$-cut of IFS on a universe $X$. Section 3 describes our main results.

Notation. Throughout this paper $J$ stands for a complete closed chain with order type 
$\theta, 1$ as a largest element, 0 as the least one and unary, involutive, order-reversing operator $n$. We note by:

$$
J^{0}=J-\{0\}, J^{1}=J-\{1\} \text { and } J^{01}=J-\{0,1\} .
$$

\section{Preliminaries}

In this section, we give some notations, definitions and results on which our work in this paper is based.

\subsection{Lukasiewicz-Moisil algebra}

Definition 1 (Moisil $[13,14])$ An involutive $\theta$-valued Lukasiewicz-Moisil algebra or simply an involutive $L-M_{\theta}$ algebra for short is a 5-tuple $\left(L, J,\left(\varphi_{\alpha}\right)_{\alpha \in J^{0}},\left(\Psi_{\alpha}\right)_{\alpha \in J^{1}}, N\right)$, where:

1) $(L, \vee, \wedge, 0,1)$ is a distributive lattice with a smallest element 0 and a greatest element $1,(C(L)$ is the Boolean sublattice of complemented elements of $L)$;

2) $N$ is an order reversing involution in $L$ satisfying for all $x \in C(L)$, $N(x)=\bar{x},(\bar{x}$ is the complement element of $x)$;

3) $\left(\varphi_{\alpha}\right)_{\alpha \in J^{0}}$ is a family of morphisms $L \longrightarrow C(L)$ in which the following properties are fulfilled : $\forall \alpha, \beta \in J^{0}$ :

(i) $\varphi_{\alpha}(0)=0$ and $\varphi_{\alpha}(1)=1$

(ii) If $\alpha \leq \beta$ then $\varphi_{\beta} \leq \varphi_{\alpha}$

(iii) $\varphi_{\alpha} \circ \varphi_{\beta}=\varphi_{\beta}$

(iv) If $\varphi_{\alpha}(x)=\varphi_{\alpha}(y)$ for all $\alpha \in J^{0}$ then $x=y$ (Moisil's determination principle);

4) $\left(\Psi_{\alpha}\right)_{\alpha \in J^{1}}$ is a family of morphisms $L \longrightarrow C(L)$ in which the following properties are fulfilled:

(i) $\Psi_{\alpha} \leq \varphi_{\alpha}, \quad$ for all $\alpha \in J^{01}$

(ii) $\varphi_{\alpha} N=N \Psi_{n \alpha}, \quad$ for all $\alpha \in J^{0}$

We note that 3-ii, 4-i and 4-ii imply $\Psi_{\beta} \leq \Psi_{\alpha}$, for all $0 \leq \alpha<\beta<1$

We use the same symbols $\leq, 0,1$ for the partial order, least and greatest elements of $J$, respectively, and for those of the algebra $L$. We shall freely note $x \leq y$ or $y \geq x$ in the sequel.

Example 1 Let $(B, \wedge, \vee, 0,1)$ be a boolean algebra. The set

$$
D(B)=\{f / f: J \longrightarrow B, i \leq j \Rightarrow f(j) \leq f(i)\}
$$

of all decreasing functions from $J$ to $B$ can be made into an involutive $L-M_{\theta}$ algebra where the operations of the lattice $(D(B), \wedge, \vee, 0,1)$ are defined for all $i \in J$ by 


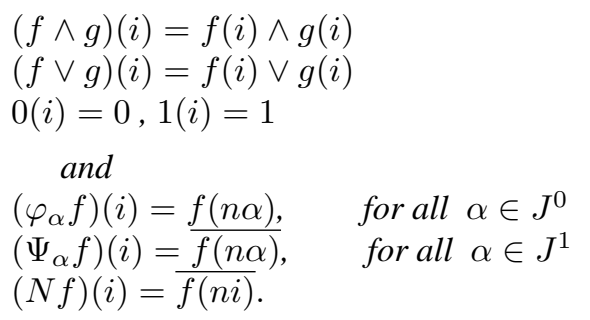

Definition 2 A filter of a lattice $(L, \vee, \wedge, 0,1)$ is a nonempty subset $F$ of $L$ such that:

(i) If $x, y \in F$, then $x \wedge y \in F$

(ii) If $x \in F$ and $x \leq y$, then $y \in F$.

A filter $F$ of $L$ is called proper filtre if it does not contains 0 .

Definition 3 Let $L$ be a lattice and $U$ a proper filter in $L$. Then $U$ is said to be a maximal filter (more usually known as an ultrafilter) if the only filter properly containing $U$ is $L$.

We will need the following Theorem and Lemma.

Theorem 1 (see [19], Theorem 1)

Let $B$ be a Boolean algebra and $X$ be the set of its ultrafilters. Then, $B$ is isomorphic to $\mathcal{P}(X)$, the embedding monomorphism being given by $\sigma(x)=\{U \in X / x \in U\}$.

Lemma 1 (see [10], Lemma 3.2)

Let $\left(L, J,\left(\varphi_{\alpha}\right)_{\alpha \in J^{0}},\left(\Psi_{\alpha}\right)_{\alpha \in J^{1}}, N\right)$ be an involutive $L-M_{\theta}$ algebra. Then

$$
\left(\Psi_{\beta} \geq \varphi_{\alpha}, \text { for all } 0<\beta<\alpha<1\right)
$$

Definition 4 Two involutive $L-M_{\theta}$ algebras $\left(L, J,\left(\varphi_{\alpha}\right)_{\alpha \in J^{0}},\left(\Psi_{\alpha}\right)_{\alpha \in J^{1}}, N\right)$ and $\left(L^{\prime}, J,\left(\varphi_{\alpha}^{\prime}\right)_{\alpha \in J^{0}},\left(\Psi_{\alpha}^{\prime}\right)_{\alpha \in J^{1}}, N^{\prime}\right)$ are said to be homomorphic if there exists a morphism $f: L \longrightarrow L^{\prime}$, such that:

(i) $f \circ \varphi_{\alpha}=\varphi_{\alpha}^{\prime} \circ f$, for all $\alpha \in J^{0}$;

(ii) $f \circ N=N^{\prime} \circ f$.

If $f$ is a monomorphism, we say that $L$ can be embedded into $L^{\prime}$.

We note that (i) and (ii) implies $f \circ \Psi_{\alpha}=\Psi_{\alpha}^{\prime} \circ f, \forall \alpha \in J^{1}$. 


\subsection{Intuitionistic fuzzy subsets}

From what was explained above, the aim of this paper is the elaboration of a representation theory of involutive $\theta$-valued Lukasiewicz-Moisil algebras, the concept of intuitionistic fuzzy subsets playing the role that the notion of field of sets plays for the representation of Boolean algebras. To provide this representation, we recall the intuitionistic fuzzy subset on a universe $X$ as an object of the form $A=\left\{\left\langle x, \mu_{A}(x), \nu_{A}(x)\right\rangle / x \in X\right\}$, where $\mu_{A}(x) \in J$ is called the degree of membership of $x$ in $A, \nu_{A}(x) \in J$ is called the degree of non-membership of $x$ in $A$ and satisfy the following condition:

$$
\mu_{A}(x) \leq n \nu_{A}(x), \quad \text { for all } x \in X .
$$

The class of intuitionistic fuzzy subsets (briefly IFSs) on a universe $X$ will be denoted by $\operatorname{IF}(X)$.

For every two IFSs $A$ and $B$ several operations are defined (see $[3,4,5,20])$. Here we shall present only those which are related to the present paper:

An intuitionistic fuzzy subset $A$ defined in the universe $X$ is empty if

$$
\mu_{A}(x)=0 \text { and } \nu_{A}(x)=1, \quad \text { for all } x \in X .
$$

If $A, B \in I F(X), A$ is an intuitionistic fuzzy subset of $B$ if

$$
\mu_{A}(x) \leq \mu_{B}(x) \text { and } \nu_{A}(x) \geq \nu_{B}(x), \quad \text { for all } x \in X .
$$

$A, B$ are equal intuitionistic fuzzy subsets if

$$
\mu_{A}(x)=\mu_{B}(x) \text { and } \nu_{A}(x)=\nu_{B}(x), \quad \text { for all } x \in X \text {. }
$$

The union and the intersection are defined as follows:

$$
\begin{aligned}
& A \cup B=\left\{\left\langle x, \sup \left(\mu_{A}(x), \mu_{B}(x)\right), \inf \left(\nu_{A}(x), \nu_{B}(x)\right)\right\rangle / x \in X\right\} . \\
& A \cap B=\left\{\left\langle x, \inf \left(\mu_{A}(x), \mu_{B}(x)\right), \sup \left(\nu_{A}(x), \nu_{B}(x)\right)\right\rangle / x \in X\right\} .
\end{aligned}
$$

Example 2 Let $X=\{1,2,3,4,5\}$ and $J$ be the real interval $[0,1]$ equipped with an order-reversing involution $n$ defined for all $\alpha \in J$ by n $\alpha=1-\alpha$. Thus, the following subsets

$$
\begin{gathered}
A=\{\langle 1,0.2,0.6\rangle\langle 2,0.3,0.6\rangle\langle 3,0.5,0.5\rangle\langle 4,0.5,0.3\rangle\langle 5,0.9,0.1\rangle\} \text { and } \\
B=\{\langle 1,0.6,0.2\rangle\langle 2,0.8,0.2\rangle\langle 3,0.8,0.1\rangle\langle 4,0.9,0.1\rangle\langle 5,1,0\rangle\}
\end{gathered}
$$


are intuitionistic fuzzy subsets on the universe X.

$$
\begin{gathered}
A \cup B=\{\langle 1,0.6,0.2\rangle\langle 2,0.8,0.2\rangle\langle 3,0.8,0.1\rangle\langle 4,0.9,0.1\rangle\langle 5,1,0\rangle\}, \\
A \cap B=\{\langle 1,0.2,0.6\rangle\langle 2,0.3,0.6\rangle\langle 3,0.5,0.5\rangle\langle 4,0.5,0.3\rangle\langle 5,0.9,0.1\rangle\} .
\end{gathered}
$$

The structure $(I F(X), \cup, \cap)$ of all intuitionistic fuzzy subsets is a distributive lattice with a smallest element $\emptyset$ and greatest element $X$. Since $J$ is equipped with an unary, involutive, order-reversing operator $n$, the intuitionistic fuzzy complementation is the order-reversing involution $\tilde{n}$ on $I F(X)$ defined, for all $A \in I F(X)$ by:

$$
\tilde{n}(A)=\left\{\left\langle x, \mu_{\tilde{n} A}(x), \nu_{\tilde{n} A}(x)\right\rangle / x \in X\right\}=\left\{\left\langle x, n \mu_{A}(x), n \nu_{A}(x)\right\rangle / x \in X\right\} .
$$

To show that the set $I F(X)$ of all intuitionistic fuzzy subsets over $X$ admits a structure of involutive $\theta$-valued L-M algebra, we define, for each $\alpha \in J^{0}$, the intuitionistic weak $\alpha$-cut as a mapping $N_{\alpha}: I F(X) \rightarrow \mathcal{P}(X)$ defined by:

$$
N_{\alpha}(A)=\left\{x \in X / \mu_{A}(x) \geq \alpha \text { and } \nu_{A}(x) \leq n \alpha\right\}
$$

and for each $\alpha \in J^{1}$, the intuitionistic strong $\alpha$-cut as a mapping $N_{\alpha}^{\prime}: I F(X) \rightarrow \mathcal{P}(X)$ defined by:

$$
N_{\alpha}^{\prime}(A)=\left\{x \in X / \mu_{A}(x)>\alpha \text { and } \nu_{A}(x)<n \alpha\right\} .
$$

\section{Representation theorems}

In this section, we will present the relationships between the involutive $L-M_{\theta}$ algebra and algebra of IFSs. But, we prove the two following lemmas which are the key results of our paper.

Lemma 2 The families of maps $\left(N_{\alpha} / \alpha \in J^{0}\right)$ and $\left(N_{\alpha}^{\prime} / \alpha \in J^{1}\right)$ satisfy the following conditions:

a) $N_{\alpha}(\emptyset)=\emptyset, N_{\alpha}(X)=X$.

b) If $\alpha \leq \beta$ then $N_{\beta} \leq N_{\alpha}$.

c) $N_{\alpha} \circ N_{\beta}=N_{\beta}$.

d) If $A, B \in I F(X)$ and for every $\alpha \in J^{0}, N_{\alpha}(A)=N_{\alpha}(B)$, then $A=B$ (Moisil's determination principle).

e) $N_{\alpha}^{\prime} \leq N_{\alpha}$, for all $\alpha \in J^{01}$. 
f) If for all $A \in \operatorname{IF}(X),\left(\mu_{A}(x)=n \nu_{A}(x)\right.$, for all $\left.x \in X\right)$ then $N_{\alpha} \tilde{n}=\tilde{n} N_{n \alpha}^{\prime}$ for all $\alpha \in J^{0}$.

Proof. a)

$$
\begin{aligned}
N_{\alpha}(\emptyset) & =\left\{x \in X / \mu_{\emptyset}(x) \geq \alpha \text { and } \nu_{\emptyset}(x) \leq n \alpha\right\} \\
& =\{x \in X / 0 \geq \alpha \text { and } 1 \leq n \alpha\}=\emptyset . \\
N_{\alpha}(X) & =\left\{x \in X / \mu_{X}(x) \geq \alpha \text { and } \nu_{X}(x) \leq n \alpha\right\} \\
& =\{x \in X / 1 \geq \alpha \text { and } 0 \leq n \alpha\}=X .
\end{aligned}
$$

b) Let $\alpha, \beta \in J^{0}$ such that $\alpha \leq \beta$. Then we have

$$
\begin{aligned}
N_{\beta}(A) & =\left\{x \in X / \mu_{A}(x) \geq \beta \text { and } \nu_{A}(x) \leq n \beta\right\} \\
& \subseteq\left\{x \in X / \mu_{A}(x) \geq \alpha \text { and } \nu_{A}(x) \leq n \alpha\right\}
\end{aligned}
$$

Hence, $N_{\beta} \leq N_{\alpha}$.

c) $N_{\alpha} \circ N_{\beta}(A)=N_{\alpha}\left(N_{\beta}(A)\right)=N_{\beta}(A)$ because $N_{\beta}(A) \in P(X)$.

d) If $N_{\alpha}(A)=N_{\alpha}(B)$ for all $\alpha \in J^{0}$, then

$\left\{x \in X / \mu_{A}(x) \geq \alpha\right.$ and $\left.\nu_{A}(x) \leq n \alpha\right\} \quad=\quad\left\{x \in X / \mu_{B}(x) \geq \alpha\right.$ and $\left.\nu_{B}(x) \leq n \alpha\right\}$,

for all $\alpha \in J^{0}$. Consequently $\mu_{A}(x)=\mu_{B}(x)$ and $\nu_{A}(x)=\nu_{B}(x)$, for all $x \in X$.

Therefore, $A=B$.

e)

$$
\begin{aligned}
N_{\alpha}^{\prime}(A) & =\left\{x \in X / \mu_{A}(x)>\alpha \text { and } \nu_{A}(x)<n \alpha\right\} \\
& \subseteq\left\{x \in X / \mu_{A}(x) \geq \alpha \text { and } \nu_{A}(x) \leq n \alpha\right\}=N_{\alpha}(A) .
\end{aligned}
$$

Hence, $N_{\alpha}^{\prime} \leq N_{\alpha}$, for all $\alpha \in J^{01}$. 
f)

$$
\begin{aligned}
N_{\alpha} \tilde{n}(A) & =\left\{x \in X / \mu_{\tilde{n} A}(x) \geq \alpha \text { and } \nu_{\tilde{n} A}(x) \leq n \alpha\right\} \\
& =\left\{x \in X / n \mu_{A}(x) \geq \alpha \text { and } n \nu_{A}(x) \leq n \alpha\right\} \\
& =\left\{x \in X / \mu_{A}(x) \leq n \alpha \text { and } \nu_{A}(x) \geq \alpha\right\}
\end{aligned}
$$

Since $\left(\mu_{A}(x)=n \nu_{A}(x)\right.$, for all $\left.x \in X\right)$, then

$$
\begin{aligned}
N_{\alpha} \tilde{n}(A) & =\left\{x \in X / \mu_{A}(x) \leq n \alpha\right\} \\
& =\overline{\left\{x \in X / \mu_{A}(x)>n \alpha\right\}},
\end{aligned}
$$

where

$$
\overline{\left\{x \in X / \mu_{A}(x)>n \alpha\right\}}=X-\left\{x \in X / \mu_{A}(x) \leq n \alpha\right\} .
$$

From the fact that: if $A \in \mathcal{P}(X), \tilde{n}(A)=\bar{A}(\bar{A}=X-A)$, it is easy to see that:

$$
\begin{aligned}
N_{\alpha} \tilde{n}(A) & =\overline{N_{n \alpha}^{\prime}(A)} \\
& =\tilde{n} N_{n \alpha}^{\prime}(A), \forall \alpha \in J^{0}
\end{aligned}
$$

For any sublattice $F$ of $I F(X)$, containing $\emptyset$ and $X$, closed under the intuitionistic fuzzy complementation $\tilde{n}$, closed under the intuitionistic weak $\alpha$-cut $N_{\alpha}$, for all $\alpha \in J^{0}$ and under the intuitionistic strong $\alpha$-cut $N_{\alpha}^{\prime}$, for all $\alpha \in J^{1}$, the sequence $\left(F, J,\left(N_{\alpha}\right)_{\alpha \in J^{0}},\left(N_{\alpha}^{\prime}\right)_{\alpha \in J^{1}}, \tilde{n}\right)$ is called an involutive $\theta$-valued intuitionistic fuzzy algebra.

Lemma 3 Let $\left(L, J,\left(\varphi_{\alpha}\right)_{\alpha \in J^{0}},\left(\Psi_{\alpha}\right)_{\alpha \in J^{1}}, N\right)$ be an involutive $L-M_{\theta}$ algebra and $U$ be a proper filter. Then

$$
\sup \left\{\alpha \in J^{0} / \varphi_{\alpha}(x) \in U\right\}=\sup \left\{\beta \in J^{1} / \Psi_{\beta}(x) \in U\right\}, \text { for all } x \in L .
$$

Proof. Let be $\alpha \in J^{0}$ and $\beta \in J^{1}$, since $J$ is a totaly ordered set, we have that either $\alpha \leq \beta$ or $\alpha>\beta$. On the one hand, if $\alpha \leq \beta$, then $\Psi_{\beta} \leq \Psi_{\alpha} \leq \varphi_{\alpha}$. Thus, if $\Psi_{\beta}(x) \in U$ then $\varphi_{\alpha}(x) \in U$, for all $x \in L$. Hence,

$$
\sup \left\{\beta \in J^{1} / \Psi_{\beta}(x) \in U\right\} \geq \sup \left\{\alpha \in J^{0} / \varphi_{\alpha}(x) \in U\right\}
$$

On the other hand, if $\alpha>\beta$, then from the inequality $\left(\Psi_{\beta} \geq \varphi_{\alpha}\right.$, for all $0<\beta<$ $\alpha<1$ ) given by Lemma 1 it follows that for all $x \in L, \varphi_{\alpha}(x) \in U$ implies $\Psi_{\beta}(x) \in U$. Hence,

$$
\sup \left\{\beta \in J^{1} / \Psi_{\beta}(x) \in U\right\} \leq \sup \left\{\alpha \in J^{0} / \varphi_{\alpha}(x) \in U\right\} \text { for all } x \in L
$$


It is clear from (1) and (2) that:

$$
\sup \left\{\alpha \in J^{0} / \varphi_{\alpha}(x) \in U\right\}=\sup \left\{\beta \in J^{1} / \Psi_{\beta}(x) \in U\right\} .
$$

Theorem 2 Every involutive $\theta$-valued intuitionistic fuzzy algebra $\left(F, J,\left(N_{\alpha}\right)_{\alpha \in J^{0}},\left(N_{\alpha}^{\prime}\right)_{\alpha \in J^{1}}, \tilde{n}\right)$ satisfying: For all $A \in F,\left(\mu_{A}(x)=n \nu_{A}(x)\right.$, for all $\left.x \in X\right)$ is an involutive $\theta$-valued Lukasiewicz-Moisil algebra.

Proof. Easily follows from Lemma 2.

Theorem 3 Every involutive $L-M_{\theta}$ algebra $\left(L, J,\left(\varphi_{\alpha}\right)_{\alpha \in J^{0}},\left(\Psi_{\alpha}\right)_{\alpha \in J^{1}}, N\right)$ can be embedded in an algebra of the form $\left(I F(X), J,\left(N_{\alpha}\right)_{\alpha \in J^{0}},\left(N_{\alpha}^{\prime}\right)_{\alpha \in J^{1}}, \tilde{n}\right)$, where $X$ is the set of ultrafilters of $C(L)$.

Proof. Let $f: L \longrightarrow I F(X)$ be the mapping defined, for all $x \in L$ by:

$$
f(x)=\left\{\left\langle U, \mu_{f(x)}(U), \nu_{f(x)}(U)\right\rangle / U \in X\right\}
$$

where $\forall U \in X$,

$$
\mu_{f(x)}(U)=\sup \left\{\alpha \in J^{0} / \varphi_{\alpha}(x) \in U\right\}
$$

and

$$
\nu_{f(x)}(U)=n \sup \left\{\alpha \in J^{0} / \varphi_{\alpha}(x) \in U\right\}=n \mu_{f(x)}(U) .
$$

Since $J$ is a complete chain, we obtain that both $\mu_{f(x)}(U)$ and $\nu_{f(x)}(U) \in J$. Also, $\mu_{f(x)}(U)=n \nu_{f(x)}(U), \quad \forall U \in X$ implies that $\left(F(L), J,\left(N_{\alpha}\right)_{\alpha \in J^{0}},\left(N_{\alpha}^{\prime}\right)_{\alpha \in J^{1}}, \tilde{n}\right)$ is an involutive $L-M_{\theta}$ algebra.

Now we prove that $f$ is a monomorphism of involutive $L-M_{\theta}$ algebras.

$$
\begin{aligned}
& f(0)=\left\{\left\langle U, \mu_{f(0)}(U), \nu_{f(0)}(U)\right\rangle / U \in X\right\} \text { such that: } \\
& \qquad \begin{aligned}
\mu_{f(0)}(U) & =\sup \left\{\alpha \in J^{0} / \varphi_{\alpha}(0) \in U\right\} \\
& =\sup \left\{\alpha \in J^{0} / 0 \in U\right\} \\
& =\sup \{\emptyset\}=0,
\end{aligned}
\end{aligned}
$$

and

$$
\nu_{f(0)}(U)=n \mu_{f(0)}(U)=n 0=1
$$

Hence, $f(0)=\emptyset$.

$f(1)=\left\{\left\langle U, \mu_{f(1)}(U), \nu_{f(1)}(U)\right\rangle / U \in X\right\}$ such that: 


$$
\begin{aligned}
\mu_{f(1)}(U) & =\sup \left\{\alpha \in J^{0} / \varphi_{\alpha}(1) \in U\right\} \\
& =\sup \left\{\alpha \in J^{0} / 1 \in U\right\} \\
& =\sup \left\{J^{0}\right\}=1
\end{aligned}
$$

and

$$
\nu_{f(1)}(U)=n \mu_{f(1)}(U)=n 1=0 .
$$

Hence, $f(1)=X$.

$$
\begin{aligned}
f(x \vee y)=\{\langle U, & \left.\left.\mu_{f(x \vee y)}(U), \nu_{f(x \vee y)}(U)\right\rangle / U \in X\right\} \text { such that: } \\
\mu_{f(x \vee y)}(U) & =\sup \left\{\alpha \in J^{0} / \varphi_{\alpha}(x \vee y) \in U\right\} \\
& =\sup \left\{\alpha \in J^{0} / \varphi_{\alpha}(x) \vee \varphi_{\alpha}(y) \in U\right\} \\
& =\sup \left\{\alpha \in J^{0} / \varphi_{\alpha}(x) \in U \vee \varphi_{\alpha}(y) \in U\right\} \\
& =\sup \left\{\alpha \in J^{0} / \varphi_{\alpha}(x) \in U\right\} \vee \sup \left\{\alpha \in J^{0} / \varphi_{\alpha}(y) \in U\right\} \\
& =\mu_{f(x)}(U) \vee \mu_{f(y)}(U),
\end{aligned}
$$

and

$$
\begin{aligned}
\nu_{f(x \vee y)}(U) & =n \mu_{f(x \vee y)}(U) \\
& =n\left(\mu_{f(x)}(U) \vee \mu_{f(y)}(U)\right) \\
& =n \mu_{f(x)}(U) \wedge n \mu_{f(y)}(U) \\
& =\nu_{f(x)}(U) \wedge \nu_{f(y)}(U) .
\end{aligned}
$$

Hence, $f(x \vee y)=f(x) \cup f(y)$.

Similarly, $f(x \wedge y)=f(x) \cap f(y)$.

$f$ is injective since for $x, y \in L$ holds $f(x)=f(x)$, then $\forall U \in X$,

$$
\left\{\left\langle U, \mu_{f(x)}(U), \nu_{f(x)}(U)\right\rangle / U \in X\right\}=\left\{\left\langle U, \mu_{f(y)}(U), \nu_{f(y)}(U)\right\rangle / U \in X\right\}
$$

According to the definition of equal intuitionistic fuzzy subsets we obtain:

$$
\mu_{f(x)}(U)=\mu_{f(y)}(U) \text { and } \nu_{f(x)}(U)=\nu_{f(y)}(U) .
$$


Thus,

$$
\sup \left\{\alpha \in J^{0} / \varphi_{\alpha}(x) \in U\right\}=\sup \left\{\alpha \in J^{0} / \varphi_{\alpha}(y) \in U\right\} .
$$

This implies that:

$$
\left\{\alpha \in J^{0} / \varphi_{\alpha}(x) \in U\right\}=\left\{\alpha \in J^{0} / \varphi_{\alpha}(y) \in U\right\} .
$$

Hence, for all $\alpha \in J^{0}, \varphi_{\alpha}(x) \in U$ iff $\varphi_{\alpha}(y) \in U$. Consequently

$$
\left\{U \in X / \varphi_{\alpha}(x) \in U\right\}=\left\{U \in X / \varphi_{\alpha}(y) \in U\right\} .
$$

Applying Theorem 1 yields $\varphi_{\alpha}(x)=\varphi_{\alpha}(y)$ for all $\alpha \in J^{0}$, and then $x=y$ (Moisil's determination principle).

Hence, $f$ is a lattice monomorphism.

The next step is to show that $f \circ \varphi_{\alpha}=N_{\alpha} \circ f$, for all $\alpha \in J^{0}$ and $f \circ N=\tilde{n} \circ f$.

1) $f \circ \varphi_{\alpha}=N_{\alpha} \circ f$,

$$
\begin{aligned}
f \circ \varphi_{\alpha}(x) & =f\left(\varphi_{\alpha}(x)\right) \\
& =\left\{\left\langle U, \mu_{f\left(\varphi_{\alpha}(x)\right)}(U), \nu_{f\left(\varphi_{\alpha}(x)\right)}(U)\right\rangle / U \in X\right\}
\end{aligned}
$$

such that:

$$
\begin{aligned}
\mu_{f\left(\varphi_{\alpha}(x)\right)}(U) & =\sup \left\{\beta \in J^{0} / \varphi_{\beta}\left(\varphi_{\alpha}(x)\right) \in U\right\} \\
& =\sup \left\{\beta \in J^{0} / \varphi_{\alpha}(x) \in U\right\} \\
& =\left\{\begin{array}{c}
0, \text { if } \varphi_{\alpha}(x) \notin U \\
1, \text { otherwise }
\end{array}\right.
\end{aligned}
$$

and

$$
\begin{aligned}
\nu_{f\left(\varphi_{\alpha}(x)\right)}(U) & =n \mu_{f\left(\varphi_{\alpha}(x)\right)}(U) \\
& =\left\{\begin{array}{c}
1, \text { if } \varphi_{\alpha}(x) \notin U \\
0, \text { otherwise }
\end{array}\right.
\end{aligned}
$$

Therefore,

$$
f \circ \varphi_{\alpha}(x)=\left\{\begin{array}{c}
\emptyset, \text { if } \varphi_{\alpha}(x) \notin U \\
X, \text { otherwise }
\end{array}=\sigma\left(\varphi_{\alpha}(x)\right) .\right.
$$




$$
\begin{aligned}
N_{\alpha} \circ f(x) & =N_{\alpha}(f(x)) \\
& =\left\{U \in X / \mu_{f(x)}(U) \geq \alpha \text { and } \nu_{f(x)}(U) \leq n \alpha\right\} \\
& =\left\{U \in X / \alpha \in\left\{\beta \in J^{0} / \varphi_{\beta}(x) \in U\right\}\right\} \\
& =\left\{U \in X / \varphi_{\alpha}(x) \in U\right\} \\
& =\sigma\left(\varphi_{\alpha}(x)\right)
\end{aligned}
$$

Hence, $f \circ \varphi_{\alpha}=N_{\alpha} \circ f$.

2) We prove that $f \circ N=\tilde{n} \circ f$. However, before doing this we need to show that

$$
\sup \left\{\alpha \in J^{0} / \Psi_{n \alpha}(x) \notin U\right\}=n \sup \left\{\alpha \in J^{1} / \Psi_{\alpha}(x) \in U\right\} .
$$

Suppose that $\sup \left\{\alpha \in J^{0} / \Psi_{n \alpha}(x) \notin U\right\} \neq n \sup \left\{\alpha \in J^{1} / \Psi_{\alpha}(x) \in U\right\}$, then there exists $\alpha \in\left\{\alpha \in J^{0} / \Psi_{n \alpha}(x) \notin U\right\}$ in which $\alpha<n \lambda$ or $\alpha>n \lambda$ such that $\lambda=\sup \left\{\alpha \in J^{1} / \Psi_{\alpha}(x) \in U\right\}$. Moreover, $n \alpha \geq \lambda$ or $n \alpha \leq \lambda$ implies that $\Psi_{n \alpha}(x) \leq \Psi_{\lambda}(x)$ or $\Psi_{n \alpha}(x) \geq \Psi_{\lambda}(x)$. Hence, $\Psi_{\lambda}(x) \notin U$ or $\Psi_{n \alpha}(x) \in U$, which is contracting with the hypothesis.

Now for all $x \in L, f \circ N(x)=f(N x)=\left\{\left\langle U, \mu_{f(N x)}(U), \nu_{f(N x)}(U)\right\rangle / U \in X\right\}$ where $\forall U \in X$,

$$
\mu_{f(N x)}(U)=\sup \left\{\alpha \in J^{0} / \varphi_{\alpha}(N x) \in U\right\},
$$

by Definition 1(4-ii) we have:

$$
\mu_{f(N x)}(U)=\sup \left\{\alpha \in J^{0} / N \Psi_{n \alpha}(x) \in U\right\}=\sup \left\{\alpha \in J^{0} / \Psi_{n \alpha}(x) \notin U\right\}
$$

From the equality (3) given above we obtain for all $x \in L$,

$$
\mu_{f(N x)}(U)=n \sup \left\{\alpha \in J^{1} / \Psi_{\alpha}(x) \in U\right\} .
$$

We know by Lemma 3 that

$$
\sup \left\{\alpha \in J^{1} / \Psi_{\alpha}(x) \in U\right\}=\sup \left\{\alpha \in J^{0} / \varphi_{\alpha}(x) \in U\right\} .
$$

Therefore,

$$
\begin{aligned}
\mu_{f(N x)}(U) & =n \sup \left\{\alpha \in J^{0} / \varphi_{\alpha}(x) \in U\right\} \\
& =n \mu_{f(x)}(U)
\end{aligned}
$$


Moreover,

$$
\nu_{f(N x)}(U)=n \mu_{f(N x)}(U)=n n \mu_{f(x)}(U)=n \nu_{f(x)}(U) .
$$

Then

$$
\begin{aligned}
f \circ N(x) & =\left\{\left\langle U, n \mu_{f(x)}(U), n \nu_{f(x)}(U)\right\rangle / U \in X\right\} \\
& =\left\{\left\langle U, \mu_{\tilde{n} f(x)}(U), \nu_{\tilde{n} f(x)}(U)\right\rangle / U \in X\right\} \\
& =\tilde{n}(f(x))
\end{aligned}
$$

Hence

$$
f \circ N=\tilde{n} \circ f
$$

\section{Conclusion}

In this paper, we have found under the condition that $J$ is a complete chain, every involutive $\theta$-valued Lukasiewicz-Moisil algebra $\left(L, J,\left(\varphi_{\alpha}\right)_{\alpha \in J^{0}},\left(\Psi_{\alpha}\right)_{\alpha \in J^{1}}, N\right)$ can be embedded in an algebra of intuitionistic fuzzy subsets on a universe $X$, where $X$ is the set of ultrafilters of $C(L)$.

Our future objective in this field is to develop a propositional logic in which the results can be used to obtain a completeness theorem for propositional many-valued calculi whose algebraic models are this kind of Lukasiewicz-Moisil algebras.

Acknowledgment. The authors wish to thank Professor A. Iorgulescu for useful remarks and suggestions concerning this paper. The authors are also grateful to the editor and referees for their useful remarks and suggestions concerning this paper.

\section{References}

[1] A. Amroune, Représentation des algèbres de Lukasiewicz $\theta$-valentes involutives par des structures floues, Bulletin for Studied and Exchanges on Fuzziness and its Applications (BUSEFAL), 43(1990), 5-11.

[2] K. T. Atanassov, Intuitionistic fuzzy sets, VII ITKR's Session, Sofia, june 1983, Deposed in Central Sci. Techn. Library of Bulg. Acad. of Sci., 1984 (in Bulgarian).

[3] K. T. Atanassov, Intuitionistic fuzzy sets, Fuzzy Sets and Systems, 20 (1986), 87-96.

[4] K. T. Atanassov and G. Gargov, Interval valued intuitionistic fuzzy sets, Fuzzy Sets and Systems, 31 (1989), 343-349. 
[5] K. T. Atanassov, Intuitionistic fuzzy sets, Physica-Verlag, Heidelberg, New york,1999.

[6] R. Biswas, On fuzzy sets and intuitionistic fuzzy sets, NIFS, 3(1997), 3-11.

[7] V. Boicescu, A. Filipoiu, G. Georgescu, and S. Rudeanu, Lukasiewicz Moisil algebras, North Holland, Elsevier, Amesterdam, New york, 1991.

[8] R. Cignoli, Algebras de Moisil, Notas de Logica Matematica, 27, Instituto de Matematica, Universidad del Sur, Bahia Blanca, 1970.

[9] J. Coulon and J. L. Coulon, Un nouveau résultat concernant la représentation d'une algèbre de Lukasiewicz involutive dans l'algèbre des parties floues d'une structure floue involutive, Rev. Roumaine Math. Pures et Appl., 38(1993), 319-326.

[10] M. De Glas, Representation of Lukasiewicz many-valued algebras, Journal of Mathematical Analysis And Applications 114 (1986), 315-327.

[11] GR. C. Moisil, Notes sur les logiques non-chrysippiennes, Ann. Sci. Univ. Jassy 27 (1941), 86-98.

[12] GR. C. Moisil, Lukasiewician algebras, Preprint, competing Center, Univ. of Bucharest, 1968.

[13] GR. C. Moisil, Essais sur les logiques non chrysippiennes, Editions de l'Academie, Bucarest 1972.

[14] GR. C. Moisil, Ensembles flous et logiques à plusieurs valeurs, Univ. de Montréal, Mai 1973.

[15] GR. C. Moisil, Sur l'emploi des mathématiques dans les sciences de l'homme Accd. Naz. Lincei. Contributi del Centro Linceo Interdisciplinare di Sci. Mat. e loro Appl., 17(1976).

[16] C. V. Negoita and D. A. Ralescu, Applications of fuzzy sets to system analysis, Birkhauser Verlag, Basel, 1975.

[17] D. Ponasse, Algèbres floues et algèbres de Lukasiewicz, Rev. Roumaine Math. Pures et Appl., 23(1978), 103-111.

[18] S. Rudeanu, On Lukasiewicz Moisil algebras of fuzzy sets, Studia Logica, 52 (1993), 95-111.

[19] M. H. Stone, The theory of representation for Boolean algebras, Trans. Amer. Math. Soc., 40(1936), 37-111.

[20] Guo-Jun Wang and Ying-Yutte, Intuitionistic fuzzy sets and L-fuzzy sets, Fuzzy Sets and Systems, 110(2000), 271-274.

[21] L. A. Zadeh, Fuzzy sets, Inform. and Control, 8 (1965), 338-353. 